\title{
Attractors for a class of semi-linear degenerate parabolic equations
}

\author{
Alessia E. KogoJ And Stefanie Sonner
}

Abstract. We consider degenerate parabolic equations of the form

$$
\begin{aligned}
\partial_{t} u & =\Delta_{\lambda} u+f(u) \\
\left.u\right|_{\partial \Omega} & =0,\left.u\right|_{t=0}=u_{0}
\end{aligned}
$$

in a bounded domain $\Omega \subset \mathbb{R}^{N}$, where $\Delta_{\lambda}$ is a subelliptic operator of the type

$$
\Delta_{\lambda}:=\sum_{i=1}^{N} \partial_{x_{i}}\left(\lambda_{i}^{2} \partial_{x_{i}}\right), \quad \lambda=\left(\lambda_{1}, \ldots, \lambda_{N}\right) .
$$

We prove global existence of solutions and characterize their longtime behavior. In particular, we show the existence and finite fractal dimension of the global attractor of the generated semigroup and the convergence of solutions to an equilibrium solution when time tends to infinity.

\section{Introduction}

We study the global existence and longtime behavior of solutions of the problem

$$
\begin{array}{rlrl}
\partial_{t} u(x, t) & =\Delta_{\lambda} u(x, t)+f(u(x, t)) & x \in \Omega, t>0, \\
u(x, t) & =0 & x \in \partial \Omega, t \geq 0, \\
u(x, 0) & =u_{0}(x) & x \in \Omega,
\end{array}
$$

in a bounded domain $\Omega \subset \mathbb{R}^{N}$, where $\Delta_{\lambda}$ is the following degenerate elliptic operator

$$
\Delta_{\lambda}:=\sum_{i=1}^{N} \partial_{x_{i}}\left(\lambda_{i}^{2} \partial_{x_{i}}\right), \quad \lambda=\left(\lambda_{1}, \ldots, \lambda_{N}\right): \mathbb{R}^{N} \rightarrow \mathbb{R}^{N} .
$$

Operators of this kind were introduced by Franchi and Lanconelli in 1982 and studied in the articles [8,9] and [10]. Recently, in [14] they were named $\Delta_{\lambda}$-Laplacian under the additional assumption that the operators are homogeneous of degree two

Mathematics Subject Classification (2000): 35B41, 35K65, 35B25, $35 \mathrm{R} 03$

Keywords: Semilinear degenerate parabolic equations, gradient semigroups, global attractors, longtime behavior of solutions.

Stefanie Sonner is funded by the ERC Advanced Grant FPT-246775 NUMERIWAVES. 
with respect to a group of dilations in $\mathbb{R}^{N}$, and existence, non-existence and regularity results for solutions of the semilinear problem

$$
\begin{aligned}
& \Delta_{\lambda} u(x)+f(u(x))=0 \quad x \in \Omega, \\
& u(x)=0 \quad x \in \partial \Omega,
\end{aligned}
$$

were obtained. The class of $\Delta_{\lambda}$-operators contains for example operators of the form

$$
\partial_{x_{1}}^{2}+\left|x_{1}\right|^{2 \alpha} \partial_{x_{2}}^{2}+\left|x_{1}\right|^{2 \beta}\left|x_{2}\right|^{2 \gamma} \partial_{x_{3}}^{2}, \quad x=\left(x_{1}, x_{2}, x_{3}\right) \in \mathbb{R}^{3},
$$

where $\alpha, \beta$ and $\gamma$ are real positive constants, and, as a particular case, the Grushin type operators (see Sect. 2.3). Problems involving Grushin type operators have been widely studied over the years, see, e.g., [15], where the subelliptic equation with critical nonlinearities was considered, and the references therein.

The existence of the global attractor for our problem (1) involving the Grushin operator was established in [3]. We extend the result for the $\Delta_{\lambda}$-Laplacian and show the finite fractal dimension of the attractor. To prove the well-posedness of Problem (1) we apply semigroup methods. In particular, we use a Poincaré type inequality and that the $\Delta_{\lambda}$-Laplacian is self-adjoint to show that $-\Delta_{\lambda}$ generates an analytic semigroup in $L^{2}(\Omega)$. The Sobolev embeddings obtained in [14] allow us to locally solve the semilinear problem under sub-critical growth restrictions on the non-linearity. The growth restrictions are determined by the homogeneous dimension $Q$ of $\mathbb{R}^{N}$ with respect to the group of dilations corresponding to the $\Delta_{\lambda}$-operator. $Q$ will play the same role as the dimension $N$ for the Laplacian in the functional setting naturally associated to $\Delta_{\lambda}$. Comparing with the classical problem for the semilinear heat equation, the degeneracy decreases the admissible growth of the nonlinearity. The global existence of solutions can be established under certain dissipativity conditions on the non-linearity. To characterize the longtime behavior of solutions of Problem (1) we apply methods from the theory of infinite dimensional dynamical systems.

The structure of our paper is as follows: In Sect. 2 we introduce the $\Delta_{\lambda}$-Laplacian, the associated functional setting and recall embedding results. We further give examples for the class of $\Delta_{\lambda}$-operators. In Sect. 3 we summarize notions and results from the theory of infinite dimensional dynamical systems that we need in the sequel. Finally, we prove the global existence of solutions for Problem (1) and characterize their longtime behavior in Sect. 4.

\section{The $\Delta_{\lambda}$-Laplacian}

\subsection{Definition and properties}

Throughout the paper $\Omega$ denotes an open bounded subset of $\mathbb{R}^{N}, N \geq 2$. As in [14], we consider operators of the form 


$$
\Delta_{\lambda}:=\sum_{i=1}^{N} \partial_{x_{i}}\left(\lambda_{i}^{2} \partial_{x_{i}}\right),
$$

where $\partial_{x_{i}}=\frac{\partial}{\partial_{x_{i}}}, i=1, \ldots, N$. The functions $\lambda_{i}: \mathbb{R}^{N} \rightarrow \mathbb{R}$ are continuous, strictly positive and of class $C^{1}$ outside the coordinate hyperplanes ${ }^{1}$ and satisfy the following properties:

(A1) $\lambda_{1}(x) \equiv 1, \lambda_{i}(x)=\lambda_{i}\left(x_{1}, \ldots, x_{i-1}\right), i=2, \ldots, N$.

(A2) For every $x \in \mathbb{R}^{N}$ the function $\lambda_{i}(x)=\lambda_{i}\left(x^{*}\right), i=1, \ldots, N$, where

$$
x^{*}=\left(\left|x_{1}\right|, \ldots,\left|x_{N}\right|\right) \text { if } x=\left(x_{1}, \ldots, x_{N}\right) .
$$

(A3) There exists a constant $\rho \geq 0$ such that

$$
0 \leq x_{k} \partial_{x_{k}} \lambda_{i}(x) \leq \rho \lambda_{i}(x) \quad \forall k \in\{1, \ldots, i-1\}, i=2, \ldots, N,
$$

and for every $x \in \mathbb{R}_{+}^{N}:=\left\{\left(x_{1}, \ldots, x_{N}\right) \in \mathbb{R}^{N}: x_{i} \geq 0 \quad \forall i=1, \ldots, N\right\}$.

(A4) There exists a group of dilations $\left(\delta_{r}\right)_{r>0}$

$$
\delta_{r}: \mathbb{R}^{N} \rightarrow \mathbb{R}^{N}, \quad \delta_{r}(x)=\delta_{r}\left(x_{1}, \ldots, x_{N}\right)=\left(r^{\varepsilon_{1}} x_{1}, \ldots, r^{\varepsilon_{N}} x_{N}\right),
$$

where $1 \leq \varepsilon_{1} \leq \varepsilon_{2} \leq \cdots \leq \varepsilon_{N}$, such that $\lambda_{i}$ is $\delta_{r}$-homogeneous of degree $\varepsilon_{i}-1$, i.e.,

$$
\lambda_{i}\left(\delta_{r}(x)\right)=r^{\varepsilon_{i}-1} \lambda_{i}(x), \quad \forall x \in \mathbb{R}^{N}, r>0, i=1, \ldots, N .
$$

This implies that the operator $\Delta_{\lambda}$ is $\delta_{r}$-homogeneous of degree two, i.e.,

$$
\Delta_{\lambda}\left(u\left(\delta_{r}(x)\right)\right)=r^{2}\left(\Delta_{\lambda} u\right)\left(\delta_{r}(x)\right) \quad \forall u \in C^{\infty}\left(\mathbb{R}^{N}\right) .
$$

We will denote by $Q$ the homogeneous dimension of $\mathbb{R}^{N}$ with respect to the group of dilations $\left(\delta_{r}\right)_{r>0}$, i.e.,

$$
Q:=\varepsilon_{1}+\cdots+\varepsilon_{N} .
$$

$Q$ plays a crucial role, both, in the geometry and the functional setting naturally associated to the operator $\Delta_{\lambda}$.

We remark that assumption (A1) allows us to write the operator in the form

$$
\Delta_{\lambda}=\sum_{i=1}^{N}\left(\lambda_{i} \partial_{x_{i}}\right)^{2} .
$$

If the functions $\lambda_{i}, i=1, \ldots, N$, are smooth, the hypotheses (1) and (4) imply that our $\Delta_{\lambda}$-Laplacian belongs to the general class of operators studied by Hörmander in [13] and that it is hypoelliptic (see Remark 1.3, [14]).

${ }^{1} \lambda_{i}>0$ in $\mathbb{R}^{N} \backslash \Pi$, where $\Pi=\left\{\left(x_{1}, \ldots, x_{N}\right) \in \mathbb{R}^{N}: \prod_{i=1}^{N} x_{i}=0\right\}$. 
2.2. Functional setting and embedding properties

For a function of class $C^{1}$ we define

$$
\nabla_{\lambda} u=\left(\lambda_{1} \partial_{x_{1}} u, \ldots, \lambda_{1} \partial_{x_{N}} u\right), \quad\left|\nabla_{\lambda} u\right|^{2}:=\sum_{i=1}^{N}\left|\lambda_{i} \partial_{x_{i}} u\right|^{2},
$$

and denote by $\stackrel{\circ}{W}_{\lambda}^{1,2}(\Omega)$ the closure of $C_{0}^{1}(\Omega)$ with respect to the norm

$$
\|u\|_{\stackrel{\circ}{\lambda}_{\lambda}^{1,2}(\Omega)}:=\left(\int_{\Omega}\left|\nabla_{\lambda} u(x)\right|^{2} d x\right)^{\frac{1}{2}} .
$$

From Proposition 3.2 and Theorem 3.3 in [14] we obtain the following embedding properties:

PROPOSITION 1. The embedding

$$
\stackrel{\circ}{W}_{\lambda}^{1,2}(\Omega) \hookrightarrow L^{p}(\Omega)
$$

is continuous for $p \in\left[1,2_{\lambda}^{*}\right]$ and compact for every $p \in\left[1,2_{\lambda}^{*}\right)$, where $2_{\lambda}^{*}:=\frac{2 Q}{Q-2}$.

REMARK 1. Since we assume $\lambda_{1} \equiv 1$ in $(i)$, a Poincaré type inequality can be obtained for the $\Delta_{\lambda}$-operator following the classical proof of the Poincaré inequality in Theorem 6.30, [1]:

There exists a constant $C>0$, such that

$$
\|u\|_{L^{2}(\Omega)} \leq C\|u\|_{W_{\lambda}^{1,2}(\Omega)} \quad \forall u \in C_{0}^{1}(\Omega)
$$

and the optimal constant in the inequality is attained by $\frac{1}{\sqrt{\mu_{1}}}$, where $\mu_{1}>0$ denotes the first eigenvalue of the operator $-\Delta_{\lambda}$ on $\Omega$ with homogeneous Dirichlet boundary conditions.

This inequality is in fact a particular case of the embedding results in Proposition 1.

\subsection{Examples of $\Delta_{\lambda}$-Laplacians}

In this subsection we use the following notations: We split $\mathbb{R}^{N}$ into

$$
\mathbb{R}^{N}=\mathbb{R}^{N_{1}} \times \cdots \times \mathbb{R}^{N_{k}},
$$

and write

$$
x=\left(x^{(1)}, \ldots, x^{(k)}\right), \quad x^{(i)}=\left(x_{1}^{(i)}, \ldots, x_{N_{i}}^{(i)}\right) \in \mathbb{R}^{N_{i}}, \quad i=1, \ldots, k .
$$

We denote the classical Laplace operator in $\mathbb{R}^{N_{i}}$ by

$$
\Delta_{x^{(i)}}=\sum_{j=1}^{N_{i}} \partial_{x_{j}^{(i)}}^{2}
$$


and write our operators in the form

$$
\Delta_{\lambda}=\left(\lambda^{(1)}\right)^{2} \Delta_{x^{(1)}}+\cdots+\left(\lambda^{(k)}\right)^{2} \Delta_{x^{(k)}} \text { in } \mathbb{R}^{N}=\mathbb{R}^{N_{1}} \times \cdots \times \mathbb{R}^{N_{k}},
$$

where

$$
\lambda=\left(\lambda^{(1)}, \ldots, \lambda^{(k)}\right), \quad \lambda^{(i)}=\left(\lambda_{1}^{(i)}, \ldots, \lambda_{N_{i}}^{(i)}\right)
$$

and the functions $\lambda^{(i)}$ are continuous in $\mathbb{R}^{N_{i}}, i=1, \ldots, k$.

EXAMPLE 1. Let $\alpha$ be a real positive constant and $k=2$. We consider the operator

$$
\Delta_{\lambda}=\Delta_{x^{(1)}}+\left|x^{(1)}\right|^{2 \alpha} \Delta_{x^{(2)}}
$$

where $\lambda=\left(\lambda^{(1)}, \lambda^{(2)}\right)$, with $\lambda_{j}^{(1)}(x)=1, j=1, \ldots, N_{1}$ and $\lambda_{j}^{(2)}(x)=\left|x^{(1)}\right|^{\alpha}$, $j=1, \ldots, N_{2}$. Using the relation (3) we find our group of dilations in (A4)

$$
\delta_{r}\left(x^{(1)}, x^{(2)}\right)=\left(r x^{(1)}, r^{\alpha+1} x^{(2)}\right),
$$

and the homogenous dimension with respect to $\left(\delta_{r}\right)_{r>0}$ is $Q=N_{1}+N_{2}(\alpha+1)$. Operators of this form are commonly called Grushin type operators. ${ }^{2}$

More generally, for a given multi-index $\alpha=\left(\alpha_{1}, \ldots, \alpha_{k-1}\right)$ with real constants $\alpha_{j} \geq 0, j=1, \ldots, k-1$, we define

$$
\Delta_{\lambda}=\Delta_{x^{(1)}}+\left|x^{(1)}\right|^{2 \alpha_{1}} \Delta_{x^{(2)}}+\cdots+\left|x^{(k-1)}\right|^{2 \alpha_{k-1}} \Delta_{x^{(k)}} .
$$

Then, in our notation $\lambda=\left(\lambda^{(1)}, \ldots, \lambda^{(k)}\right)$ with

$$
\begin{array}{rlrl}
\lambda_{j}^{(1)}(x) & \equiv 1, & j & =1, \ldots, N_{1}, \\
\lambda_{j}^{(i)}(x) & =\left|x^{(i-1)}\right|^{\alpha_{i-1}} & i & =2, \ldots, k, j=1, \ldots, N_{i},
\end{array}
$$

and the group of dilations such that $\lambda$ satisfies (3) is given by

$$
\delta_{r}\left(x^{(1)}, \ldots, x^{(k)}\right)=\left(r^{\varepsilon_{1}} x^{(1)}, \ldots, r^{\varepsilon_{k}} x^{(k)}\right)
$$

with $\varepsilon_{1}=1$ and $\varepsilon_{i}=\alpha_{i-1} \varepsilon_{i-1}+1$ for $i=2, \ldots, k$. In particular, if $\alpha_{1}=\cdots=$ $\alpha_{k-1}=\alpha$, the dilations become

$$
\delta_{r}\left(x^{(1)}, \ldots, x^{(k)}\right)=\left(r x^{(1)}, r^{\alpha+1} x^{(2)}, \ldots, r^{\alpha^{k-1}+\cdots+\alpha+1} x^{(k)}\right) .
$$

EXAMPLE 2. Let $\alpha, \beta$ and $\gamma$ be nonnegative real constants. For the operator

$$
\Delta_{\lambda}=\Delta_{x^{(1)}}+\left|x^{(1)}\right|^{2 \alpha} \Delta_{x^{(2)}}+\left|x^{(1)}\right|^{2 \beta}\left|x^{(2)}\right|^{2 \gamma} \Delta_{x^{(3)}},
$$

\footnotetext{
${ }^{2}$ In 1970 Grushin [11] studied these operators assuming that $\alpha$ is a positive integer and provided a complete characterization of the hypoellipticity for such operators when lower terms with complex coefficients are added.
} 
where $\lambda=\left(\lambda^{(1)}, \lambda^{(2)}, \lambda^{(3)}\right)$ with

$$
\begin{aligned}
\lambda_{j}^{(1)}(x) & \equiv 1, & j & =1, \ldots, N_{1}, \\
\lambda_{j}^{(2)}(x) & =\left|x^{(1)}\right|^{\alpha}, & j & =1, \ldots, N_{2}, \\
\lambda_{j}^{(3)}(x) & =\left|x^{(1)}\right|^{\beta}\left|x^{(2)}\right|^{\gamma}, & j & =1, \ldots, N_{3},
\end{aligned}
$$

we find the group of dilations

$$
\delta_{r}\left(x^{(1)}, x^{(2)}, x^{(3)}\right)=\left(r x^{(1)}, r^{\alpha+1} x^{(2)}, r^{\beta+(\alpha+1) \gamma+1} x^{(3)}\right) .
$$

Similarly, for operators of the form

$$
\begin{aligned}
\Delta_{\lambda}= & \Delta_{x^{(1)}}+\left|x^{(1)}\right|^{2 \alpha_{1,1}} \Delta_{x^{(2)}}+\left|x^{(1)}\right|^{2 \alpha_{2,1}}\left|x^{(2)}\right|^{2 \alpha_{2,2}} \Delta_{x^{(3)}} \\
& +\cdots+\left(\prod_{i=1}^{k-1}\left|x^{(i)}\right|^{2 \alpha_{k-1, i}}\right) \Delta_{x^{(k)}}
\end{aligned}
$$

where $\alpha_{i, j} \geq 0, i=1, \ldots, k-1, j=1, \ldots, i$, are real constants, the group of dilations is given by

$$
\delta_{r}\left(x^{(1)}, \ldots, x^{(k)}\right)=\left(r^{\varepsilon_{1}} x^{(1)}, \ldots, r^{\varepsilon_{k}} x^{(k)}\right)
$$

with $\varepsilon_{1}=1$ and $\varepsilon_{j}=1+\sum_{i=1}^{j-1} \alpha_{j-1, i} \varepsilon_{i}$, for $i=2, \ldots, k$.

In particular, if $\alpha_{1,1}=\cdots=\alpha_{k-1, k-1}=\alpha$, the dilations become

$$
\delta_{r}\left(x^{(1)}, \ldots, x^{(k)}\right)=\left(r x^{(1)}, r^{\alpha+1} x^{(2)}, \ldots, r^{(\alpha+1)^{k-1}} x^{(k)}\right) .
$$

EXAMPLE 3. We assume $p_{1}, p_{2}: \mathbb{R}^{N_{1}} \longrightarrow \mathbb{R}$ are homogeneous polynomials with positive coefficients of the variables $\left|x_{1}^{(1)}\right|, \ldots,\left|x_{N_{1}}^{(1)}\right|$ and $p_{3}: \mathbb{R}^{N_{2}} \longrightarrow \mathbb{R}$ is an homogeneous polynomial with positive coefficients of the variables $\left|x_{1}^{(2)}\right|, \ldots,\left|x_{N_{2}}^{(2)}\right|$. For the operator

$$
\Delta_{\lambda}=\Delta_{x^{(1)}}+\left(p_{1}\left(x^{(1)}\right)\right)^{2} \Delta_{x^{(2)}}+\left(p_{2}\left(x^{(1)}\right)\right)^{2}\left(p_{3}\left(x^{(2)}\right)\right)^{2} \Delta_{x^{(3)}}
$$

we find the group of dilations

$$
\delta_{r}\left(x^{(1)}, x^{(2)}, x^{(3)}\right)=\left(r x^{(1)}, r^{m_{1}+1} x^{(2)}, r^{m_{2}+\left(m_{1}+1\right) m_{3}+1} x^{(3)}\right),
$$

where $m_{i}$ is the degree of the polynomial $p_{i}, i=1,2,3$.

More generally, let $\mu_{1}, \mu_{2}: \mathbb{R}^{N_{1}} \longrightarrow \mathbb{R}$ and $\mu_{3}: \mathbb{R}^{N_{2}} \longrightarrow \mathbb{R}$ be continuous functions, positive and of class $C^{1}$ outside the coordinate hyperplanes, that satisfy (A2), (A3) and

$$
\mu_{1}\left(s x^{(1)}\right)=s^{\alpha} \mu_{1}\left(x^{(1)}\right), \quad \mu_{2}\left(s x^{(1)}\right)=s^{\beta} \mu_{2}\left(x^{(1)}\right), \quad \mu_{3}\left(s x^{(2)}\right)=s^{\gamma} \mu_{3}\left(x^{(2)}\right), \quad \forall s>0,
$$


where $\alpha, \beta$ and $\gamma$ are nonnegative real constants. The group of dilations for the operator

$$
\Delta_{\lambda}=\Delta_{x^{(1)}}+\left(\mu_{1}\left(x^{(1)}\right)\right)^{2} \Delta_{x^{(2)}}+\left(\mu_{2}\left(x^{(1)}\right)\right)^{2}\left(\mu_{3}\left(x^{(2)}\right)\right)^{2} \Delta_{x^{(3)}}
$$

is given by

$$
\delta_{r}\left(x^{(1)}, x^{(2)}, x^{(3)}\right)=\left(r x^{(1)}, r^{\alpha+1} x^{(2)}, r^{\beta+(\alpha+1) \gamma+1} x^{(3)}\right) .
$$

REMARK 2. The last example generalizes Example 1.5 in [14],

$$
\Delta_{\lambda}=\Delta_{x^{(1)}}+\left(\mu\left(x^{(1)}\right)\right)^{2} \Delta_{x^{(2)}},
$$

where the authors missed to add that the function $\mu$ satisfies the hypotheses (A2) and (A3).

As they mentioned in Note 2, if $\mu\left(x^{(1)}\right)=\frac{1}{2}\left|x^{(1)}\right|$ the operator $\Delta_{\lambda}$ takes the form

$$
\Delta_{x^{(1)}}+\frac{1}{4}\left|x^{(1)}\right|^{2} \Delta_{x^{(2)}} .
$$

Furthermore, if the dimensions $N_{1}$ and $N_{2}$ verify the inequality $N_{2}<\rho\left(N_{1}\right)$, where $\rho$ is the so called Hurwitz-Radon function, then there exists a composition law $\circ$ in $\mathbb{R}^{N}$ making $\mathbb{H}_{N}:=\left(\mathbb{R}^{N}, \circ, \delta_{\lambda}\right)$ a group of Heisenberg type (see [5], Remark 3.6.7). Denoting by $\Delta_{\mathbb{H}_{N}}$ the canonical sub-Laplacian on $\mathbb{H}_{N}$, we have

$$
\left(\Delta_{x^{(1)}}+\frac{1}{4}\left|x^{(1)}\right|^{2} \Delta_{x^{(2)}}\right) u=\Delta_{\mathbb{H}_{N}} u,
$$

for every smooth function $u: \mathbb{R}^{N} \longrightarrow \mathbb{R}$ which is radially symmetric in the variable $x^{(1)}$ (see [5], p. 251).

\section{Global attractors of infinite dimensional dynamical systems: some well-known properties}

We summarize in this section notions from the theory of infinite dimensional dynamical systems and recall a general existence result for global attractors. For further details we refer to [16] or [4].

Let $S(t): V \rightarrow V, t \geq 0$, be continuous operators in a Banach space $\left(V,\|\cdot\|_{V}\right)$. We call the family $S(t), t \geq 0$, a semigroup if it satisfies the properties

$$
\begin{aligned}
S(t) \circ S(s) & =S(t+s) \quad \forall t, s \geq 0, \\
S(0) & =\mathrm{Id}, \\
(t, v) & \mapsto S(t) v \quad \text { is continuous from }[0, \infty) \times V \rightarrow V,
\end{aligned}
$$

where $\circ$ denotes the composition, and Id the identity operator in $V$. 
For a subset $B \subset V$ we define the positive orbit of $B$ by

$$
\gamma^{+}(B):=\bigcup_{t \geq 0} S(t) B,
$$

and more generally, for $\tau \geq 0$ we define the orbit of $B$ after time $\tau$ by

$$
\gamma_{\tau}^{+}(B):=\gamma^{+}(S(\tau) B)
$$

The semigroup $S(t), t \geq 0$, is asymptotically compact if for every bounded subset $B \subset V$ such that $\gamma_{\tau}^{+}(B)$ is bounded for some $\tau \geq 0$, the set $\left\{S\left(t_{n}+\tau\right) v_{n}, n \in \mathbb{N}\right\}$, is relatively compact for all sequences $v_{n}$ in $B$ and $t_{n} \geq 0$ such that $t_{n} \rightarrow \infty$ as $n \rightarrow \infty$.

We call a non-empty compact subset $\mathcal{A} \subset V$ the global attractor of the semigroup $S(t), t \geq 0$, if $\mathcal{A}$ is invariant, i.e.,

$$
S(t) \mathcal{A}=\mathcal{A} \quad \forall t \geq 0
$$

and $\mathcal{A}$ attracts every bounded subset $B \subset V$ under the action of the semigroup, i.e.,

$$
\lim _{t \rightarrow \infty} \operatorname{dist}_{H}(S(t) B, \mathcal{A})=0 .
$$

Here, $\operatorname{dist}_{H}(\cdot, \cdot)$ denotes the Hausdorff semi-distance in $V$, i.e.,

$$
\operatorname{dist}_{H}(B, A):=\sup _{b \in B} \inf _{a \in A}\|a-b\|_{V} \text { for subsets } A, B \subset V .
$$

A Lyapunov functional for the semigroup $S(t), t \geq 0$, is a continuous function $\Phi: V \rightarrow \mathbb{R}$ such that

$$
\begin{array}{ll}
\Phi(S(t) v) \leq \Phi(v) & \forall t \geq 0, \forall v \in V \\
\Phi(S(t) v)=\Phi(v) & \forall t \geq 0 \text { implies that } v \text { is an equilibrium point. }
\end{array}
$$

If $S(t), t \geq 0$, possesses a Lyapunov functional we call it a gradient semigroup. Moreover, we denote the set of equilibrium points of the semigroup $S(t), t \geq 0$, by

$$
\mathcal{E}:=\{v \in V: S(t) v=v \forall t \geq 0\},
$$

and the unstable set of $\mathcal{E}$ is

$\mathcal{W}^{u}(\mathcal{E})=\left\{v \in V: S(t) x\right.$ is defined for all $t \in \mathbb{R}, \operatorname{dist}_{H}(S(-t) v, \mathcal{E}) \rightarrow 0$ as $\left.t \rightarrow \infty\right\}$

For the proof of the following theorem about the existence of global attractors for gradient semigroups we refer to Theorem 4.6 and Proposition 2.19, [16].

THEOREM 1. Let $S(t), t \geq 0$, be an asymptotically compact gradient semigroup such that for every bounded subset $B \subset V$ there exists $\tau \geq 0$ such that the orbit $\gamma_{\tau}^{+}(B)$ is bounded. If the set of equilibrium points $\mathcal{E}$ is bounded, then the global attractor exists, is connected and $\mathcal{A}=W^{u}(\mathcal{E})$. 
The $\omega$-limit set of an element $v \in V$ is

$$
\begin{aligned}
& \omega(v)=\left\{y \in V: \exists \text { a sequence } t_{n} \geq 0, n \in \mathbb{N}, t_{n} \rightarrow \infty,\right. \text { such that } \\
&\left.S\left(t_{n}\right) v \rightarrow y \text { as } n \rightarrow \infty\right\} .
\end{aligned}
$$

The invariance principle of LaSalle (see Proposition 4.2, [16]) characterizes the longtime behavior of trajectories.

PROPOSITION 2. Let $S(t), t \geq 0$, be a gradient semigroup in $V$ with Lyapunov functional $\Phi: V \rightarrow \mathbb{R}$ and let $u \in V$. If the orbit $\gamma_{\tau}^{+}(u)$ is relatively compact in $V$ for some $\tau \geq 0$, then the limit $\lim _{t \rightarrow \infty} \Phi(S(t) u)=$ a exists and $\Phi(v)=$ a for all $v \in \omega(u)$. Moreover, $\omega(u) \subset \mathcal{E}, \mathcal{E} \neq \emptyset$ and

$$
\operatorname{dist}_{H}(S(t) u, \mathcal{E}) \rightarrow_{t \rightarrow \infty} 0 .
$$

\section{Global existence of solutions and their longtime behavior}

We consider the degenerate parabolic initial value problem

$$
\begin{array}{rlrl}
\partial_{t} u(x, t) & =\Delta_{\lambda} u(x, t)+f(u(x, t)) & x \in \Omega, t>0, \\
u(x, t) & =0 & x \in \partial \Omega, t \geq 0, \\
u(x, 0) & =u_{0}(x) & x \in \Omega,
\end{array}
$$

where $\Omega \subset \mathbb{R}^{N}$ is a bounded domain, and the initial data $u_{0} \in \stackrel{\circ}{W}_{\lambda}^{1,2}(\Omega)$.

The non-linearity $f: \mathbb{R} \rightarrow \mathbb{R}$ is locally Lipschitz continuous and satisfies the following growth restriction: There exist constants $c \geq 0$ and $0 \leq \gamma<\frac{4}{Q-2}$ such that

$$
|f(u)-f(v)| \leq c|u-v|\left(1+|u|^{\gamma}+|v|^{\gamma}\right) \quad \forall u, v \in \mathbb{R} .
$$

Moreover, to show the global existence of solutions we assume the following sign condition:

$$
\limsup _{|u| \rightarrow \infty} \frac{f(u)}{u}<\mu_{1},
$$

where $\mu_{1}>0$ denotes the first eigenvalue of the operator $-\Delta_{\lambda}$ on $\Omega$ with homogeneous Dirichlet boundary conditions.

This in particular implies that exist constants $0 \leq c_{0}<\mu_{1}$ and $c_{1} \in \mathbb{R}$ such that

$$
u f(u) \leq c_{1}|u|+c_{0} u^{2}, \quad u \in \mathbb{R} .
$$

DEFINITION 1. We call u a local weak solution of (5) if there exists $T>0$ such that

$$
u \in C\left([0, T) ; \stackrel{\circ}{W}_{\lambda}^{1,2}(\Omega)\right), \quad u(0)=u_{0}, \quad u \in C^{1}\left((0, T) ;\left(\stackrel{\circ}{W}_{\lambda}^{1,2}(\Omega)\right)^{\prime}\right),
$$


where $Y^{\prime}$ indicates the dual space of a Banach space $Y$, and $u$ satisfies the equation

$$
\frac{d}{d t}\langle u(t), v\rangle_{L^{2}(\Omega)}=-a_{\lambda}(u(t), v)+\langle f(u), v\rangle_{L^{2}(\Omega)} \forall v \in \stackrel{\circ}{W}_{\lambda}^{1,2}(\Omega), t \in(0, T) .
$$

The bilinear form $a_{\lambda}$ in $\stackrel{\circ}{W}_{\lambda}^{1,2}(\Omega)$ is defined as

$$
a_{\lambda}(u, v):=\int_{\Omega} \nabla_{\lambda} u(x) \cdot \nabla_{\lambda} v(x) d x \quad u, v \in \stackrel{\circ}{W}_{\lambda}^{1,2}(\Omega),
$$

and $\cdot$ denotes the inner product in $\mathbb{R}^{N}$.

\subsection{Local and global existence of solutions}

We consider the $\Delta_{\lambda}$-operator in $L^{2}(\Omega)$ with domain

$$
\begin{gathered}
\mathcal{D}\left(\Delta_{\lambda}\right):=\left\{u \in \stackrel{\circ}{W}_{\lambda}^{1,2}(\Omega): \exists c \geq 0 \text { such that }\left|a_{\lambda}(u, v)\right| \leq c\|v\|_{L^{2}(\Omega)} \forall v \in \stackrel{\circ}{W}_{\lambda}^{1,2}(\Omega)\right\}, \\
-\left\langle\Delta_{\lambda} u, v\right\rangle_{L^{2}(\Omega)}=a_{\lambda}(u, v) \quad \forall u \in \mathcal{D}\left(\Delta_{\lambda}\right), v \in \stackrel{\circ}{W}_{\lambda}^{1,2}(\Omega) .
\end{gathered}
$$

Since $\Omega$ is bounded and the functions $\lambda_{i}, i=1, \ldots, N$, are continuous on $\mathbb{R}^{N}$, we remark that

$$
\mathcal{D}\left(\Delta_{D}\right)=H_{0}^{1}(\Omega) \cap H^{2}(\Omega) \subset \mathcal{D}\left(\Delta_{\lambda}\right),
$$

where $\Delta_{D}$ denotes the Laplace operator in $\Omega$ with homogeneous Dirichlet boundary conditions.

PROPOSITION 3. The operator $-\Delta_{\lambda}$ generates an analytic semigroup $e^{\Delta_{\lambda} t}$, $t \geq 0$, in $L^{2}(\Omega)$.

Proof. The $\Delta_{\lambda}$-Laplacian is densely defined and self-adjoint in $L^{2}(\Omega)$. By Inequality (4), $-\Delta_{\lambda}$ is bounded from below by a positive constant $C>0$,

$$
-\int_{\Omega} \Delta_{\lambda} u(x) u(x) \mathrm{d} x=\int_{\Omega}\left|\nabla_{\lambda} u(x)\right|^{2} \mathrm{~d} x \geq C\|u\|_{L^{2}(\Omega)}^{2} \quad \forall u \in \mathcal{D}\left(-\Delta_{\lambda}\right) .
$$

which implies that $-\Delta_{\lambda}$ is sectorial (see [12], p. 19). The proposition now follows from Theorem 1.3.4 in [12].

The operator $A:=-\Delta_{\lambda}$ is positive and self-adjoint in $L^{2}(\Omega)$ and has compact inverse by Proposition 1. Consequently, there exists an orthonormal basis of $L^{2}(\Omega)$ of eigenfunctions $\psi_{j} \in \stackrel{\circ}{W}_{\lambda}^{1,2}(\Omega), j \in \mathbb{N}$, of $A$ with eigenvalues

$$
0<\mu_{1} \leq \mu_{2} \leq \ldots, \quad \mu_{j} \rightarrow \infty \text { as } j \rightarrow \infty .
$$

We denote the fractional power spaces associated to $A$ by $X^{\alpha}=\left(\mathcal{D}\left(A^{\alpha}\right),\langle\cdot, \cdot\rangle_{X^{\alpha}}\right)$, $\alpha \in \mathbb{R}$. The inner product in $X^{\alpha}$ is given by $\langle u, v\rangle_{X^{\alpha}}=\left\langle A^{\alpha} u, A^{\alpha} v\right\rangle_{X^{0}}, u, v \in \mathcal{D}\left(A^{\alpha}\right)$, where

$$
\mathcal{D}\left(A^{\alpha}\right)=\left\{\psi=\sum_{j \in \mathbb{N}} c_{j} \psi_{j}, c_{j} \in \mathbb{R} \mid \sum_{j \in \mathbb{N}} \mu_{j}^{2 \alpha} c_{j}^{2}<\infty\right\}
$$


and

$$
A^{\alpha} \psi=A^{\alpha} \sum_{j \in \mathbb{N}} c_{j} \psi_{j}=\sum_{j \in \mathbb{N}} \mu_{j}^{\alpha} c_{j} \psi_{j}
$$

In this notation,

$$
X^{1}=\mathcal{D}\left(-\Delta_{\lambda}\right), \quad X^{\frac{1}{2}}=\stackrel{\circ}{W}_{\lambda}^{1,2}(\Omega), \quad X^{0}=L^{2}(\Omega), \quad X^{-\frac{1}{2}}=\left(\stackrel{\circ}{W}_{\lambda}^{1,2}(\Omega)\right)^{\prime} .
$$

The operator $A$ in $X^{0}$ can be extended or restricted, respectively, to a positive sectorial operator in $X^{\alpha}$ with domain $X^{\alpha+1}, \alpha \in \mathbb{R}$, and the corresponding semigroups $\mathrm{e}^{-A t}, t \geq 0$, in $X^{\alpha}$ are obtained from each other by natural restrictions and extensions. Moreover, if $\beta \leq \alpha$ we have $e^{-A t}\left(X^{\beta}\right) \subset X^{\alpha}$ and

$$
\left\|\mathrm{e}^{-A t}\right\|_{\mathcal{L}\left(X^{\beta} ; X^{\alpha}\right)} \leq \frac{C_{\alpha, \beta}}{t^{\alpha-\beta}}, \quad t>0,
$$

for some constant $C_{\alpha, \beta} \geq 0$, where $\|\cdot\|_{\mathcal{L}(V ; W)}$ denotes the norm of a linear operator between the normed spaces $V$ and $W$ (e.g., see Section II.2.1. in [17] and Theorem 2.4 in [2]).

We formulate Problem (5) in the abstract form

$$
\begin{aligned}
u_{t} & =-A u+\mathbf{f}(u), \\
\left.u\right|_{t=0} & =u_{0},
\end{aligned}
$$

with initial data $u_{0} \in X^{\frac{1}{2}}$. Here, we consider the operator $A$ in $X^{-\frac{1}{2}}$ with domain $X^{\frac{1}{2}}$, which is determined by the bilinear form $a_{\lambda}$,

$$
(A u, v)_{X^{-\frac{1}{2}}}=a_{\lambda}(u, v) \quad \forall u, v \in X^{\frac{1}{2}},
$$

and $\mathbf{f}(u) \in X^{-\frac{1}{2}}$ by

$$
(\mathbf{f}(u), v)_{X^{-\frac{1}{2}}}:=\langle f(u), v\rangle_{L^{2}(\Omega)}, \quad u, v \in X^{\frac{1}{2}},
$$

where $(\cdot, \cdot)_{X^{-\frac{1}{2}}}$ denotes the dual pairing between $X^{\frac{1}{2}}$ and $X^{-\frac{1}{2}}$.

If $u$ is a local weak solution in the sense of Definition 1 , it satisfies the variation of constants formula

$$
u(t)=T_{\lambda}(t) u_{0}+\int_{0}^{t} T_{\lambda}(t-s) f(u(s)) d s \quad \forall t \in[0, T),
$$

where $T_{\lambda}(t), t \geq 0$, denotes the analytic semigroup in $X^{-\frac{1}{2}}$ generated by the operator $A$ (see Lemma 2.2.1, [6]).

From now on the letter $C$ will always denote a non-negative constant that may vary in each occurrence and from line to line. To show the existence of solutions under the growth restrictions (6) we need the following lemma. 
LEMMA 1. We assume the function $f$ satisfies the growth restriction with $\frac{2}{Q-2}<$ $\gamma<\frac{4}{Q-2}$. Then, there exists $0<\alpha<\frac{1}{2}$ such that $f: X^{\frac{1}{2}} \rightarrow X^{-\alpha}$ is locally Lipschitz. Proof. Let $p:=\frac{2_{\lambda}^{*}}{2_{\lambda}^{*}-(\gamma+1)}$, then $p \in\left(2,2_{\lambda}^{*}\right)$. The identity $E$ is a bounded linear operator from $X^{0}=L^{2}(\Omega)$ to $L^{2}(\Omega)$ and from $X^{\frac{1}{2}}$ to $L^{p_{\lambda}^{*}}(\Omega)$ by Proposition 1 . By complex interpolation we conclude that

$$
E:\left[X^{0}, X^{\frac{1}{2}}\right]_{2 \alpha}=X^{\alpha} \rightarrow\left[L^{2}(\Omega), L^{2_{\lambda}^{*}}(\Omega)\right]_{2 \alpha}=L^{p}(\Omega), \quad \frac{1}{p}=\frac{1-2 \alpha}{2}+\frac{2 \alpha}{2_{\lambda}^{*}},
$$

is linear and bounded, where $\alpha=\frac{2_{\lambda}^{*}(p-2)}{2 p\left(2 \lambda_{\lambda}^{*}-2\right)} \in\left(0, \frac{1}{2}\right)$ (see Section II.2.1. in [17], Example 7.56 in [1] and Proposition 1.3.9 in [6]).

Let now $q:=\frac{2_{\lambda}^{*}}{\gamma+1}$. Then, $q \in(1,2), \frac{1}{p}+\frac{1}{q}=1$, and by duality we obtain $L^{q}(\Omega) \hookrightarrow X^{-\alpha}$ for some $\alpha \in\left(0, \frac{1}{2}\right)$. To conclude the proof of the lemma we show that $f: X^{\frac{1}{2}} \rightarrow L^{q}(\Omega)$ is locally Lipschitz continuous. Let $u, v \in X^{\frac{1}{2}}$ be such that $\|u\|_{X^{\frac{1}{2}}},\|v\|_{X^{\frac{1}{2}}}<r$ for some $r>0$. Using the growth condition (6) and Hölder's inequality with $r=\frac{2_{\lambda}^{*}}{q \gamma}$ and $s=\frac{2 *}{q}$ we obtain

$$
\begin{aligned}
\|f(u)-f(v)\|_{L^{q}(\Omega)}^{q} & \leq C\left(\|u-v\|_{L^{q}(\Omega)}^{q}+\int_{\Omega}\left(|u-v|^{q}|u|^{q \gamma}+|u-v|^{q}|v|^{q \gamma}\right) \mathrm{d} x\right) \\
& \leq C\left(\|u-v\|_{L^{q}(\Omega)}^{q}+\|u-v\|_{L^{s q}(\Omega)}^{q}\left(\|u\|_{L^{r q \gamma}(\Omega)}^{q \gamma}+\|u\|_{L^{r q \gamma}(\Omega)}^{q \gamma}\right)\right) \\
& =C\left(\|u-v\|_{L^{q}(\Omega)}^{q}+\|u-v\|_{L^{2^{*}(\Omega)}}^{q}\left(\|u\|_{L^{2^{*}(\Omega)}}^{q \gamma}+\|u\|_{L^{2^{*}(\Omega)}}^{q \gamma}\right)\right) \\
& \leq C\|u-v\|_{X^{\frac{1}{2}}}^{q},
\end{aligned}
$$

where we used in the last step the embedding $X^{\frac{1}{2}} \hookrightarrow L^{p}(\Omega)$, for $1 \leq p \leq 2_{\lambda}^{*}$.

THEOREM 2. We assume the function $f$ satisfies the growth restrictions (6). Then, for every initial data $u_{0} \in X^{\frac{1}{2}}$ there exists a unique local weak solution of Problem (5) defined on the maximal interval of existence $[0, T)$ and

$$
u \in C\left([0, T) ; X^{\frac{1}{2}}\right) \cap C^{1}\left((0, T) ; X^{\beta}\right) \quad \forall \beta \in[-\alpha, 1-\alpha) .
$$

The solution satisfies the variation of constants formula

$$
u(t)=T_{\lambda}(t) u_{0}+\int_{0}^{t} T_{\lambda}(t-s) f(u(s)) d s,
$$

and either $T=\infty$ or, if $T<\infty$, then

$$
\limsup _{t \rightarrow T}\|u(t)\|_{X^{\frac{1}{2}}}=\infty \text {. }
$$


Proof. Without loss of generality we can assume that $f$ satisfies the growth restrictions (6) with exponent $\frac{2}{Q-2}<\gamma<\frac{4}{Q-2}$. Indeed, if $0<\gamma \leq \frac{2}{Q-2}$ we apply Young's inequality to increase the exponent in (6). Let now $\alpha \in\left(0, \frac{1}{2}\right)$ be as in Lemma 1 . If we consider the operator $A$ in $X^{-\alpha}$ with domain $X^{1-\alpha}$ in the abstract semilinear parabolic problem (9), the theorem is an immediate consequence of Lemma 1, Theorem 2.1.1 and Corollary 2.3.1 in [6].

REMARK 3. If the non-linearity satisfies the growth restrictions (6) with exponent $0 \leq \gamma \leq \frac{2}{Q-2}$, the proof of Theorem 2 simplifies and Lemma 1 is not needed. Indeed, using Hölder's inequality and the embedding result in Theorem 1 we can show that $f$ is locally Lipschitz continuous from $X^{\frac{1}{2}}$ to $X^{0}$ (see also [16] for the proof in the classical case of the semilinear heat equation). The statement of Theorem 2 then follows from Theorem 2.1.1 and Corollary 2.3.1 in [6], and

$$
u \in C\left([0, T) ; X^{\frac{1}{2}}\right) \cap C^{1}\left((0, T) ; X^{\alpha}\right) \cap C\left((0, T) ; X^{1}\right) \quad \forall \alpha \in[0,1) .
$$

To show the global existence of solutions we follow the arguments in [16] and introduce the Lyapunov functional $\Phi: X^{\frac{1}{2}} \rightarrow \mathbb{R}$

$$
\Phi(u):=\int_{\Omega}\left(\frac{1}{2}\left|\nabla_{\lambda} u(x)\right|^{2}-F(u(x))\right) \mathrm{d} x, \quad u \in X^{\frac{1}{2}},
$$

where $F(u):=\int_{0}^{u} f(s) \mathrm{d} s$ denotes the primitive of $f$. We remark that if $u$ is a local weak solution of (5), then Theorem 2 implies that $u \in C\left([0, T) ; X^{\frac{1}{2}}\right) \cap$ $C^{1}\left((0, T) ; X^{\frac{1}{2}}\right)$. Furthermore, the Lyapunov functional satisfies $\Phi(u(\cdot)) \in C([0, T)$; $\mathbb{R}) \cap C^{1}((0, T) ; \mathbb{R})$, and

$$
\frac{\mathrm{d}}{\mathrm{d} t} \Phi(u(t))=-\left\|u_{t}(t)\right\|_{L^{2}(\Omega)}^{2}<\infty, \quad t \in(0, T) .
$$

Using the growth restriction (6) we obtain

$$
\begin{aligned}
\Phi(u(t)) \leq \Phi\left(u_{0}\right) & \leq \int_{\Omega}\left(\frac{1}{2}\left|\nabla_{\lambda} u_{0}(x)\right|^{2}+\left|F\left(u_{0}(x)\right)\right|\right) \mathrm{d} x \\
& \leq C\left(1+\frac{1}{2}\left\|u_{0}\right\|_{X^{\frac{1}{2}}}^{2}+\left\|u_{0}\right\|_{L^{\gamma+2}(\Omega)}^{\gamma+2}\right) .
\end{aligned}
$$

On the other hand, the sign condition (7) and Young's inequality yield the estimate

$$
\begin{aligned}
\Phi(u(0)) \geq \Phi(u(t)) & =\int_{\Omega}\left(\frac{1}{2}\left|\nabla_{\lambda} u(x, t)\right|^{2}-F(u(x, t))\right) \mathrm{d} x \\
& \geq \frac{1}{2}\|u(t)\|_{X^{\frac{1}{2}}}^{2}-\frac{1}{2} c_{0}\|u(t)\|_{L^{2}(\Omega)}^{2}-\int_{\Omega} c_{1}|u(x, t)| \mathrm{d} x \\
& \geq \frac{1}{2}\|u(t)\|_{X^{\frac{1}{2}}}^{2}-\frac{1}{2}\left(c_{0}+\epsilon\right)\|u(t)\|_{L^{2}(\Omega)}^{2}-C_{\epsilon} \\
& \geq \frac{1}{2}\|u(t)\|_{X^{\frac{1}{2}}}^{2}\left(1-\frac{c_{0}+\epsilon}{\mu_{1}}\right)-C_{\epsilon},
\end{aligned}
$$


for small $\epsilon>0$ and some constant $C_{\epsilon} \geq 0$, where we used Poincaré's inequality in the last step. Since $c_{0}<\mu_{1}$ and $L^{\gamma+2}(\Omega) \hookrightarrow X^{\frac{1}{2}}$, the above estimates imply that the solution $u$ is uniformly bounded in time $t \in[0, T)$ in $X^{\frac{1}{2}}$, and therefore exists globally, i.e., $T=\infty$.

4.2. Existence and finite fractal dimension of the global attractor

We verified in the previous subsection that Problem (5) generates a semigroup $S_{\lambda}(t), t \geq 0$, in $X^{\frac{1}{2}}$,

$$
S_{\lambda}(t) u_{0}=u\left(t ; u_{0}\right), \quad t \geq 0,
$$

where $u\left(\cdot ; u_{0}\right) \in C\left([0, T) ; X^{\frac{1}{2}}\right) \cap C^{1}\left((0, T) ; X^{\frac{1}{2}}\right)$ denotes the unique global weak solution of (5) corresponding to initial data $u_{0} \in X^{\frac{1}{2}}$. Moreover, the semigroup $S_{\lambda}(t), t \geq 0$, is gradient with Lyapunov functional $\Phi: X^{\frac{1}{2}} \rightarrow \mathbb{R}$.

To prove the existence and finite dimensionality of the global attractor we formulate two auxiliary results.

LEMMA 2. Let $B \subset X^{\frac{1}{2}}$ be a bounded subset. Then, for every $T^{*}>0$ there exists a constant $\kappa>0$ such that

$$
\left\|S_{\lambda}\left(T^{*}\right) u-S_{\lambda}\left(T^{*}\right) v\right\|_{X^{\frac{1}{2}}} \leq \kappa\|u-v\|_{X^{0}} \quad \forall u, v \in B .
$$

Proof. As in the proof of Theorem 2 we can assume that $f$ satisfies the growth restrictions with $\frac{2}{Q-2}<\gamma<\frac{4}{Q-2}$. Given initial data $u_{0}, v_{0} \in B$, the corresponding solutions $u(t)=S_{\lambda}(t) u_{0}$ and $v(t)=S_{\lambda}(t) v_{0}$ satisfy the variation of constants formula. By Lemma 1 and (8) we obtain for the difference

$$
\begin{aligned}
\| & S_{\lambda}(t) u_{0}-S_{\lambda}(t) v_{0} \|_{X^{\frac{1}{2}}} \\
\leq & \left\|e^{-A^{\frac{1}{2}} t}\right\|_{\mathcal{L}\left(X^{0}, X^{\frac{1}{2}}\right)}\left\|u_{0}-v_{0}\right\|_{X^{0}} \\
& +\int_{0}^{t}\left\|e^{-A^{\frac{1}{2}} t}\right\|_{\mathcal{L}\left(X^{-\alpha}, X^{\frac{1}{2}}\right)}\|f(u(s))-f(v(s))\|_{X^{-\alpha}} \mathrm{d} s \\
\leq & C\left(\frac{1}{\sqrt{t}}\left\|u_{0}-v_{0}\right\|_{X^{0}}+\int_{0}^{t} \frac{1}{(t-s)^{\frac{1}{2}+\alpha}}\|f(u(s))-f(v(s))\|_{X^{-\alpha}} \mathrm{d} s\right) \\
\leq & C\left(\frac{1}{\sqrt{t}}\left\|u_{0}-v_{0}\right\|_{X^{0}}+\int_{0}^{t} \frac{1}{(t-s)^{\frac{1}{2}+\alpha}}\|u(s)-v(s)\|_{X^{\frac{1}{2}}} \mathrm{~d} s\right) .
\end{aligned}
$$

Let $T^{*}>0$, the singular Gronwall Lemma (see Lemma 1.2.9, [6]) now implies

$$
\left\|S_{\lambda}\left(T^{*}\right) u_{0}-S_{\lambda}\left(T^{*}\right) v_{0}\right\|_{X^{\frac{1}{2}}} \leq \frac{C}{\sqrt{T^{*}}}\left\|u_{0}-v_{0}\right\|_{X^{0}} .
$$


The smoothing property allows to estimate the fractal dimension of compact invariant sets. We recall that the fractal dimension of a compact set $A \subset V$ is defined as

$$
\operatorname{dim}_{f}(A)=\lim _{\epsilon \rightarrow 0} \frac{\ln \left(N_{\epsilon}^{V}(A)\right)}{-\ln (\epsilon)},
$$

where $N_{\epsilon}^{V}(A)$ denotes the minimal number of balls in $V$ with radius $\epsilon>0$ and centers in $A$ needed to cover the set $A$.

LEMMA 3. Let $V$ and $W$ be Banach spaces such that the embedding $V \hookrightarrow W$ is dense and compact, and let $S_{\lambda}(t), t \geq 0$, be a semigroup in $V$. We assume $\mathcal{A} \subset V$ is a compact invariant set and the semigroup satisfies the smoothing property: There exists $T^{*}>0$ and a constant $\kappa \geq 0$ such that

$$
\left\|S_{\lambda}\left(T^{*}\right) u-S_{\lambda}\left(T^{*}\right) v\right\|_{V} \leq \kappa\|u-v\|_{W} \quad \forall u, v \in \mathcal{A} .
$$

Then, the fractal dimension of $\mathcal{A}$ in $V$ is finite.

Proof. The statement can be deduced by slightly modifying the method developed in [7] to construct exponential attractors for semigroups.

Finally, we prove the existence and finite fractal dimension of the global attractor for the semigroup $S_{\lambda}(t), t \geq 0$, and show that all solutions of (5) converge to the set of equilibrium solutions

$$
\mathcal{E}=\left\{u \in X^{\frac{1}{2}}: \Delta_{\lambda} u+f(u)=0\right\}
$$

when time $t$ tends to infinity. The existence of equilibrium solutions was obtained in Theorem 3.1, [14].

THEOREM 3. The semigroup $S_{\lambda}(t), t \geq 0$, possesses a global attractor $\mathcal{A}$ in $\stackrel{\circ}{W}_{\lambda}^{1,2}(\Omega)$, and its fractal dimension is finite. Furthermore, $\mathcal{A}$ is connected,

$$
\mathcal{A}=W^{u}(\mathcal{E})
$$

for every initial data $u_{0} \in X^{\frac{1}{2}}$ we have $\omega\left(u_{0}\right) \subset \mathcal{E}$, and in particular,

$$
\lim _{t \rightarrow \infty} \operatorname{dist}_{H}\left(S_{\lambda}(t) u_{0}, \mathcal{E}\right)=0 .
$$

Proof. Existence of the Global Attractor: We have shown that the semigroup $S_{\lambda}(t), t \geq$ 0 , is gradient and orbits of bounded sets are bounded. To apply Theorem 1 it remains to prove that the set of equilibria is bounded in $X^{\frac{1}{2}}$ and that $S_{\lambda}(t), t \geq 0$, is asymptotically compact. Let $u \in \mathcal{E}$. Multiplying the equation by $u$ and using Young's and Poincaré's inequality we obtain

$$
\begin{aligned}
0 & =-\|u\|_{X^{\frac{1}{2}}}^{2}+\int_{\Omega} f(u(x)) u(x) \mathrm{d} x \leq-\|u\|_{X^{\frac{1}{2}}}^{2}+\int_{\Omega}\left(c_{1}|u(x)|+\frac{c_{0}}{2}|u(x)|^{2}\right) \mathrm{d} x \\
& \leq-\|u\|_{X^{\frac{1}{2}}}^{2}\left(1-\frac{c_{0}+\epsilon}{\mu_{1}}\right)+C_{\epsilon},
\end{aligned}
$$


for $\epsilon>0$ and some constant $C_{\epsilon} \geq 0$. Since $c_{0}<\mu_{1}$ the estimate implies that the set $\mathcal{E}$ is bounded in $X^{\frac{1}{2}}$.

To prove the asymptotic compactness of $S_{\lambda}(t), t \geq 0$, we assume the set $B \subset X^{\frac{1}{2}}$ is bounded. Let $T>0$ and $x_{n} \in B, t_{n} \geq 0, n \in \mathbb{N}$, be sequences such that $t_{n} \rightarrow \infty$ as $n \rightarrow \infty$. Since orbits of bounded sets are bounded in $X^{\frac{1}{2}}$, the set $\left\{S_{\lambda}\left(t_{n}-T\right) x_{n}: t_{n} \geq T, n \in \mathbb{N}\right\}$ is bounded in $X^{\frac{1}{2}}$ and consequently, there exists a subsequence $v_{k}:=S_{\lambda}\left(t_{n_{k}}-T\right) x_{n_{k}}$ converging weakly to $v$ in $X^{\frac{1}{2}}$ and strongly to $v$ in $X^{0}$. Applying Lemma 2 we obtain

$$
\left\|S_{\lambda}(T) v_{k}-S_{\lambda}(T) v\right\|_{X^{\frac{1}{2}}} \leq \kappa\left\|v_{k}-v\right\|_{X^{0}}
$$

which implies that $S_{\lambda}(T) v_{k}=S_{\lambda}\left(t_{n_{k}}\right) v_{n_{k}}$ converges to $S_{\lambda}(T) v$ in $X^{\frac{1}{2}}$, and shows the asymptotic compactness of the semigroup $S_{\lambda}(t), t \geq 0$.

Convergence to Stationary States: Let $u_{0} \in X^{\frac{1}{2}}$. We deduce the last statement of the theorem from the invariance principle of LaSalle (see Sect. 3). It suffices to show that there exists $\tau \geq 0$ such that $\gamma_{\tau}^{+}\left(u_{0}\right)$ is relatively compact in $X^{\frac{1}{2}}$. Let $\tau>0$, then

$$
\gamma_{\tau}^{+}\left(u_{0}\right)=\bigcup_{t \geq 0} S_{\lambda}(\tau) S_{\lambda}(t) u_{0}=S_{\lambda}(\tau) \gamma^{+}\left(u_{0}\right) \text {. }
$$

Furthermore, if $x_{n}, n \in \mathbb{N}$, is a sequence in $\gamma_{\tau}^{+}\left(u_{0}\right)$, then

$$
x_{n}=S_{\lambda}(\tau) y_{n} \quad \text { with } y_{n} \in \gamma^{+}\left(u_{0}\right)
$$

Since the orbit $\gamma^{+}\left(u_{0}\right)$ is bounded in $X^{\frac{1}{2}}$ there exists a subsequence $y_{n_{k}}$ converging weakly to an element $y$ in $X^{\frac{1}{2}}$ and strongly to $y$ in $X^{0}$. We again apply Lemma 2 to conclude that the sequence $S_{\lambda}(\tau) y_{n_{k}}$ converges to $S_{\lambda}(\tau) y$ in $X^{\frac{1}{2}}$, which proves the precompactness of the orbit $\gamma_{\tau}^{+}\left(u_{0}\right)$.

Fractal Dimension of the Global Attractor: The global attractor $\mathcal{A}$ is compact and invariant, and the semigroup $S_{\lambda}(t), t \geq 0$, satisfies the smoothing property in $\mathcal{A}$. Lemma 3 applied to the semigroup $S_{\lambda}(t), t \geq 0$, with $V=X^{\frac{1}{2}}$ and $W=X^{0}$ implies the finite fractal dimension of $\mathcal{A}$.

\section{Acknowledgments}

We are grateful to Prof. Enrique Zuazua for bringing our attention to this problem and would like to thank the referees for their valuable comments and remarks that greatly improved the presentation of our results.

\section{REFERENCES}

[1] Adams, R.A., Fournier, J.J.F.: Sobolev Spaces. 2nd edition, Academic Press (2003)

[2] Amann, H.: On abstract parabolic fundamental solutions. J. Math. Soc. Japan 39, 93-116 (1987) 
[3] Anh, C.T., Hung, P.Q., Ke, T.D., Phong, T.T.: Global attractor for a semilinear parabolic equation involving the Grushin operator. Electron. J. Differential Equations 2008, 1-11 (2008)

[4] Babin, A.V., Vishik, M.I.: Attractors of Evolution Equations. North-Holland, Amsterdam (1992)

[5] Bonfiglioli, A., Lanconelli, E., Uguzzoni, F.: Stratified Lie Groups and Potential Theory for their sub-Laplacians. Springer-Verlag, Berlin, Heidelberg (2007)

[6] Cholewa, J.W., Dlotko, J.: Global Attractors in Abstract Parabolic Problems. Cambridge University Press, New York (2000)

[7] Efendiev, M.A., Miranville, A., Zelik, S.: Exponential attractors and finite-dimensional reduction for nonautonomous dynamical systems. Proc. Roy. Soc. Edinburgh 135, 703-730 (2005)

[8] Franchi, B., Lanconelli, E.: Une métrique associée à une classe d'opérateurs elliptiques dégénérés. Conference on linear partial and pseudodifferential operators (Torino, 1982), Rend. Sem. Mat. Univ. Politec. Torino 1983, Special Issue, 105-114 (1984)

[9] Franchi, B., Lanconelli, E.: An embedding theorem for Sobolev spaces related to non-smooth vectors fields and Harnack inequality. Comm. Partial Differential Equations 9, 1237-1264 (1984)

[10] Franchi, B., Lanconelli, E.: Hölder regularity theorem for a class of nonuniformly elliptic operators with measurable coefficients. Ann. Sc. Norm. Sup. Pisa Cl. Sci. 10, 523-541 (1983)

[11] Grushin, V.V.: On a class of hypoelliptic operators. Math. USSR Sbornic 12, 458-476 (1970)

[12] Henry, D.: Geometric Theory of Semilinear Parabolic Equations. Lecture Notes in Mathematics, Springer, Berlin, Heidelberg, New York (1981).

[13] Hörmander, L.: Hypoelliptic second order differential equations. Acta Math. 119, 147-171 (1967)

[14] Kogoj, A., Lanconelli, E.: On semilinear $\Delta_{\lambda}$-Laplace equation. Nonlinear Anal. 75, 4637-4649 (2012)

[15] Monti, R., Morbidelli, D.: Kelvin transform for Grushin operators and critical semilinear equations. Duke Math. J., 167-202 (2006)

[16] Raugel, G.: Global Attractors in Partial Differential Equations. Handbook of Dynamical Systems, Vol. 2, Elsevier (2002)

[17] Temam, R.: Infinite-Dimensional Dynamical Systems in Mechanics and Physics. Applied Mathematical Sciences 68, 2nd edition, Springer, New York (1997)

Alessia E. Kogoj and Stefanie Sonner

BCAM Basque Center for

Applied Mathematics,

Mazarredo 14,

48009 Bilbao, Basque Country,

Spain,

E-mail: ssonner@bcamath.org

Alessia E. Kogoj

E-mail:akogoj@bcamath.org 